Europe's Journal of Psychology 1/2009

www.ejop.org

\title{
Beliefs about Mental Illness among University Students in Egypt
}

\author{
Hani Hamed Dessoki, MD \\ Assistant Professor of Psychiatry and Acting Head, \\ Department of Psychiatry at the University of Beni Suef
}

Tamer M.S. Hifnawy MD

Lecturer of Public Health,

Faculty of Medicine Beni Suef University

\begin{abstract}
Objective: To detect differences in attitudes towards psychiatric illness, regarding its nature, cause, different ways of therapy, possibility of cure and the effect on the society in two areas from different socioeconomic classes in Egypt. Method: A survey study using self administered questionnaire, to detect culture differences between students of Cairo and Beni Suef universities "Beni Suef is one of upper Egypt governorates". All participating students were subjected to Familial Socioeconomic status scale, Beliefs about Psychiatric Illness in the Arab Culture Scale. Results: $69.8 \%$ students were females. The majority of Cairo University students were from higher socioeconomic classes (average $41 \%$ and above average $32.5 \%$ ). However, the majority of Beni Suef University students were form low and below average class (13.6\%, $49 \%$ respectively). Cairo University students showed higher positive attitude regarding Beliefs about Psychiatric Illness including the nature of psychiatric illness, psychiatric etiology, psychiatric management, psychiatric cure, and effect on the family than Beni Suef University students $(p<.001)$. Females in both groups showed lower positive attitude on Attitudes Towards Psychiatric Illness in the Arab Culture Scale than males ( $\mathrm{P}<.001)$. Conclusion: Beliefs about mental illness are highly affected by low socioeconomic status, upbringing way and beliefs in Egypt. Public education about psychiatric illnesses is highly beneficial for mental and public health professionals.
\end{abstract}

Key words: Beliefs, Mental Illness, University students, Egypt 


\section{Introduction}

Worldwide, mental and behavioral disorders are common, affecting more than $25 \%$ of all people at some time during their lives. They are also universal, affecting people of all countries and societies, regardless of age, gender and income. The point prevalence of mental illness in the adult population at any given time is about $10 \%$. Similarly, around $20 \%$ of all patients seen by primary health care providers have one or more mental health disorders [1]. Throughout the world, there is an increasing awareness of mental illness as a significant cause of morbidity [2].

The role of the community in the prevention and care of the mentally handicapped has now been widely acknowledged and is regarded as the most appropriate basis for the development of mental health programs. Several studies have shown that knowledge of public attitude to mental illness and its treatment is a vitally important prerequisite to the realization of successful community-based programs [3].

The recognition of mental disorder also depends on a careful evaluation of the norms, beliefs and customs within the individual's culture environment. Furthermore, community attitude and beliefs play a role in determining help-seeking behavior and successful treatment of the mentally ill. Unarguably, ignorance and stigma prevent the mentally ill from seeking appropriate help [4].

The relationship between the culture and the stigma attached to an illness is rather complex. It is probably even more complicated in the developing countries. It appears that different illnesses have different status attached to them. Although, some are medicalised and stigmatised, others are not. It has also been suggested that, in most of these societies some supernatural, religious, moralistic, and magical approaches to illness and behaviour exist. This can complicate the issue further [5]. People tend to have strong beliefs about the mentally ill, and many of these concepts are based on prevailing local systems of belief [6].

In Egypt as elsewhere, one of the most commonly cited reasons for the under-use of available psychiatric services by the lay-public is the notion of stigma. Stigma is frequently blamed for the cultural incompatibility of western- based mental health programs in certain context, for the continued reliance on traditional healers and the failure of certain non -western countries (and immigrants from the same) to fall in the step with the dominant psychiatric paradigm [7]. 
Researchers have most often assessed stigma associated with mental illness by surveying the public about attitudes toward "mental patients" or "persons with mental illness," terms that likely evoke images of chronic psychopathology. Consequently, it is unclear whether evidence of stigma is indicative of prejudice toward all mental illness or only its more severe forms. Although some studies have focused on the stigma associated with specific disorders [8].

People's beliefs regarding mental illness should not only be known, but the purpose of their beliefs should be understood. Such attitudes and beliefs about mental illness can only be studied within a culture context [9].

There are marked culture variations in the illness behavior of psychiatric patients. Illness behavior -as defined by Mechanic (1962) - describes how symptoms are perceived, evaluated and acted upon. Social factors are involved not only in the cognitive schemas of their symptomatology but also in the choice of different alternatives among courses of how to deal with these symptoms, i.e. the meaning and seriousness of psychiatric symptoms are deeply embedded in the patients' culture assumptions [10].

To date there is no research on public attitudes towards mental illness from Beni Suef Governorate, a culturally distinct part of the country, by having different customs and traditions. This paper is therefore one of the first to report findings related to attitudinal research on mental illness from Beni Suef Governorate.

We aimed to detect differences in attitudes towards psychiatric illness, regarding its nature, cause, different ways of therapy, possibility of cure and the effect on the society in a wide range of different culture areas in Egypt. Also, to detect the effect of socioeconomic status on these beliefs.

\section{Subjects and Methods}

This study was conducted from February to April 2007 in both Cairo and Beni Suef University, as a survey study of University students to screen for assessing differences in attitudes towards psychiatric illness, regarding its nature, cause, different ways of therapy, possibility of cure and the effect on the society. 750 self administered questionnaire were distributed to all students attending Arabic language courses in both faculty Arts in Cairo and Beni Suef Universities and enrolled in the third and the fourth academic year and they were not subjected to any courses related to psychology throughout their academic years in both faculties. Five hundred 
questionnaires were distributed in Cairo University and 250 questionnaire were distributed in Beni Suef University.

Our response rate was 480 (96\%) and 243 (97.2\%) students from both Faculty of Arts Cairo University and Faculty of Arts Beni Suef University respectively. After being informed of their rights as research participant, all students volunteered to complete the research survey in class.

All students were subjected to the following:

1- Short sheet which includes sociodemographic data (age, sex, education, marital status, residence), family history and past history.

2- Familial Sociodeconomic Status Scale [11]. This scale had been proved to be reliable and valid. It can help the user to determine the socioeconomic level for the Egyptian family. It depends mainly on the monthly income per person within the family, father's occupation and education. The following equation was used:

$w=2.259+x(1.016)+y(0.886)+z(0.622)$

$\mathrm{w}=$ Socioeconomic status .

$\mathrm{x}=$ the monthly income per person within the family.

$y=$ father's occupation.

$z=$ father's education.

According to it the socioeconomic status are classified into:

Very low $=(48-72)$, low $=(73-96)$, below average $=(97-120)$, average $=(121-144)$, above average $=(145-168)$, high $=(169-192)$, very high $=(193-216)$.

3- Beliefs about Psychiatric Illness in the Arab Culture Scale [12]. It is a 40-statements scale that includes 5 subscales to which the subject agrees, disagrees or is equivocal. These subscales are the nature of psychiatric illness, possible causes of psychiatric illness, management of psychiatric illness, possibility of cure from psychiatric illness and the effect on the family life. Validity of this scale was done by Internal consistency estimation by calculating the correlation coefficient between the Psychological Beliefs total and each one of the subscales and between the subscales themselves. Correlation coefficients were $0.46,0.73,0.57,0.65$ and 0.55 when measuring the association between the Psychological Beliefs total and psychiatric illness, etiology, management, cure and effect on family subscales. All of them showed significance level $<0.01^{* *}$. Scale reliability was assessed on 100 students from Secondary schools and Universities, the Cronbach's alpha and interclass correlation coefficient was 0.64 for the Psychological Beliefs total and 0.68, $0.52,0.66,0.66,0.91$ for the psychiatric illness, etiology, management, cure and 
effect on family subcales respectively and all of them showed significance level $<0.01^{* *}[12]$. These results suggest that our tool is valid and reliable.

All scales were applied in Arabic language.

Statistical analysis

Statistical analysis was done using the Statistical Package for Social Sciences (SPSS ver.15). To summarize the data, the number of observations and percentages were used for qualitative variables, the mean and standard deviation for quantitative variables. Chi-square test for comparison of percentages. Kruskal-Wallis and MannWhitney tests as non parametric tests for ordinal data and analysis of variance (ANOVA) for continuous data. The threshold of significance is fixed at the $5 \%$ level.

Results

I- Sociodemographic and clinical Data:

1- Age:

The age of students in both groups was 22 - 24ys.

2- Gender, Family history \& Past history:

\begin{tabular}{|c|c|c|c|c|c|c|}
\hline & & & $\begin{array}{c}\text { Beni Suef } \\
\text { University } \\
\text { students } \\
(\mathrm{No}=243)\end{array}$ & $\begin{array}{c}\text { Cairo } \\
\text { University } \\
\text { students } \\
(\mathrm{No}=480)\end{array}$ & $\begin{array}{l}\text { Total } \\
(\mathrm{No}= \\
723)\end{array}$ & $p$ \\
\hline \multirow[t]{4}{*}{ Gender } & \multirow[t]{2}{*}{ Female } & No. & 189 & 316 & 505 & \multirow{4}{*}{.001} \\
\hline & & $\%$ & 77.8 & 65.8 & 69.8 & \\
\hline & \multirow[t]{2}{*}{ Male } & No. & 54 & 164 & 218 & \\
\hline & & $\%$ & 22.2 & 34.2 & 30.2 & \\
\hline \multirow{4}{*}{$\begin{array}{l}\text { Family } \\
\text { history }\end{array}$} & \multirow[t]{2}{*}{ +ve F.H } & No. & 12 & 8 & 20 & \multirow{4}{*}{.016} \\
\hline & & $\%$ & 4.9 & 1.7 & 2.8 & \\
\hline & \multirow[t]{2}{*}{-ve F.H } & No. & 231 & 472 & 703 & \\
\hline & & $\%$ & 95.1 & 98.3 & 97.2 & \\
\hline \multirow{4}{*}{$\begin{array}{c}\text { Past } \\
\text { History }\end{array}$} & \multirow[t]{2}{*}{ +ve P.H } & No. & 7 & 5 & 12 & \multirow{4}{*}{$\begin{array}{r}.118 \\
\text { N.S }\end{array}$} \\
\hline & & $\%$ & 2.9 & 1 & 1.7 & \\
\hline & \multirow{2}{*}{$\begin{array}{l}\text {-ve } \\
\text { P.H }\end{array}$} & No. & 236 & 475 & 711 & \\
\hline & & $\%$ & 97.1 & 99 & 98.3 & \\
\hline
\end{tabular}

Table 1: Distribution of Gender, Family history \& Past history in both Groups 
II- Psychometric Data:

1- Familial Sociodeconomic Status Scale:

\begin{tabular}{|c|c|c|c|c|c|}
\hline & & $\begin{array}{l}\text { Beni Suef } \\
\text { University } \\
\text { students } \\
(\text { No= 243) }\end{array}$ & $\begin{array}{c}\text { Cairo } \\
\text { University } \\
\text { students } \\
(\mathrm{No}=480)\end{array}$ & $\begin{array}{c}\text { Total } \\
(\mathrm{No}=723)\end{array}$ & $p$ \\
\hline \multirow[t]{2}{*}{ Very low } & No. & 6 & 0 & 6 & \multirow{10}{*}{$<.001$} \\
\hline & $\%$ & 2.5 & 0 & 0.8 & \\
\hline \multirow[t]{2}{*}{ Low } & No. & 33 & 32 & 65 & \\
\hline & $\%$ & 13.6 & 6.7 & 9.0 & \\
\hline \multirow{2}{*}{$\begin{array}{c}\text { Below } \\
\text { average }\end{array}$} & No. & 119 & 95 & 214 & \\
\hline & $\%$ & 49.0 & 19.8 & 29.6 & \\
\hline \multirow[t]{2}{*}{ Average } & No. & 80 & 197 & 277 & \\
\hline & $\%$ & 32.9 & 41.0 & 38.3 & \\
\hline \multirow{2}{*}{$\begin{array}{c}\text { Above } \\
\text { average }\end{array}$} & No. & 5 & 156 & 161 & \\
\hline & $\%$ & 2.1 & 32.5 & 22.3 & \\
\hline
\end{tabular}

Table 2: Distribution of Familial Sociodeconomic Status Scale in both Groups

2- Beliefs about Psychiatric Illness in the Arab Culture Scale:

\begin{tabular}{|c|c|c|c|c|c|}
\hline \multirow{2}{*}{} & \multicolumn{2}{|c|}{$\begin{array}{r}\text { Beni Suef University } \\
\text { students } \\
\text { (No= 243) }\end{array}$} & \multicolumn{2}{c|}{$\begin{array}{r}\text { Cairo University } \\
\text { students } \\
\text { (No= 480) }\end{array}$} & P \\
\cline { 2 - 5 } & Mean & S.D. & Mean & S.D. & \\
\hline $\begin{array}{c}\text { Psychological } \\
\text { Beliefs Iotal }\end{array}$ & 53.08 & \pm 8.96 & 61.04 & \pm 5.86 & $<.001$ \\
\hline $\begin{array}{c}\text { Psychiatric illness } \\
\text { Psychiatric etiology }\end{array}$ & 11.63 & \pm 3.30 & 14.35 & \pm 2.20 & $<.001$ \\
\hline $\begin{array}{c}\text { Psychiatric } \\
\text { management }\end{array}$ & 9.82 & \pm 1.41 & 10.31 & \pm 1.41 & $<.001$ \\
\hline Psychiatric cure & 9.72 & \pm 1.90 & 10.67 & \pm 1.61 & $<.001$ \\
\hline Effect on the family & 10.76 & \pm 1.22 & 11.76 & \pm 1.35 & $<.001$ \\
\hline
\end{tabular}

Table 3a: Beliefs about Psychiatric Illness in both Groups 
b- Comparison between Females and Males regarding Beliefs about Psychiatric Illness Scale:

\begin{tabular}{|c|c|c|c|c|c|}
\hline \multirow{2}{*}{} & \multicolumn{2}{|c|}{$\begin{array}{c}\text { Female } \\
\text { (No= 505) }\end{array}$} & \multicolumn{2}{c|}{$\begin{array}{c}\text { Male } \\
\text { (No= 218) }\end{array}$} & P \\
\cline { 2 - 5 } & Mean & S.D. & Mean & S.D. & \\
\hline $\begin{array}{c}\text { Nature of } \\
\text { psychiatric illness }\end{array}$ & 13.30 & \pm 2.83 & 13.74 & \pm 2.84 & $\begin{array}{c}.062 \\
\text { N.S }\end{array}$ \\
\hline $\begin{array}{c}\text { Causes of } \\
\text { psychiatric illness }\end{array}$ & 13.38 & \pm 3.32 & 13.77 & \pm 3.01 & $\begin{array}{c}.118 \\
\text { N.S }\end{array}$ \\
\hline $\begin{array}{c}\text { Psychiatric } \\
\text { management }\end{array}$ & 9.86 & \pm 1.29 & 10.42 & \pm 1.60 & $<.001$ \\
\hline Psychiatric cure & 10.21 & \pm 1.63 & 10.68 & \pm 2.03 & .001 \\
\hline Effect on the family & 10.99 & \pm 1.22 & 11.17 & \pm 1.70 & $\begin{array}{c}.086 \\
\text { N.S }\end{array}$ \\
\hline
\end{tabular}

Table 3b: Beliefs about Psychiatric Illness among Females and Males in both Groups

c- Comparison between those who have and don't have family history regarding Beliefs about Psychiatric Illness Scale:

\begin{tabular}{|c|c|c|c|c|c|}
\hline \multirow{2}{*}{} & \multicolumn{2}{|c|}{$\begin{array}{c}\text { Positive F.H } \\
\text { (No= 20) }\end{array}$} & \multicolumn{2}{c|}{$\begin{array}{c}\text { Negative F.H } \\
\text { (No= 703) }\end{array}$} & p \\
\cline { 2 - 5 } & Mean & S.D. & Mean & S.D. & \\
\hline $\begin{array}{c}\text { Nature of } \\
\text { psychiatric illness }\end{array}$ & 14.80 & \pm 3.12 & 13.39 & \pm 2.91 & \\
\hline $\begin{array}{c}\text { Causes of } \\
\text { psychiatric illness }\end{array}$ & 14.95 & \pm 3.01 & 13.46 & \pm 3.17 & .034 \\
\hline $\begin{array}{c}\text { Psychiatric } \\
\text { management }\end{array}$ & 11.25 & \pm 2.75 & 9.99 & \pm 1.36 & $<.001$ \\
\hline Psychiatric cure & 12.00 & \pm 2.90 & 10.30 & \pm 1.71 & $<.001$ \\
\hline Effect on the family & 12.60 & \pm 2.39 & 11.00 & \pm 1.26 & $<.001$ \\
\hline
\end{tabular}

Table 3c: Beliefs about Psychiatric Illness among those who have and don't have family history in both Groups 
d- Comparison between those who have and don't have past history regarding Beliefs about Psychiatric Illness in the Arab Culture Scale:

\begin{tabular}{|c|c|c|c|c|c|}
\hline \multirow{2}{*}{} & \multicolumn{2}{|c|}{$\begin{array}{c}\text { Positive P.H } \\
\text { (No= 12) }\end{array}$} & \multicolumn{2}{c|}{$\begin{array}{c}\text { Negative P.H } \\
\text { (No= 711) }\end{array}$} & \\
\cline { 2 - 5 } & Mean & S.D. & Mean & S.D. & \\
\hline $\begin{array}{c}\text { Nature of } \\
\text { psychiatric illness }\end{array}$ & 15.25 & \pm 2.45 & 13.40 & \pm 2.92 & \multirow{2}{*}{030} \\
\hline $\begin{array}{c}\text { Causes of } \\
\text { psychiatric illness }\end{array}$ & 15.83 & \pm 1.89 & 13.46 & \pm 3.17 & \multirow{2}{*}{010} \\
\hline $\begin{array}{c}\text { Psychiatric } \\
\text { management }\end{array}$ & 11.33 & \pm 2.81 & 10.00 & \pm 1.37 & .001 \\
\hline $\begin{array}{c}\text { Psychiatric cure } \\
\text { Esfect on the family }\end{array}$ & 13.08 & \pm 2.87 & 10.31 & \pm 1.72 & $<.001$ \\
\hline
\end{tabular}

Table 3d: Beliefs about Psychiatric Illness among those who have and don't have past history in both Groups

III- Distribution of Responses of both groups on each Item of the Beliefs about Psychiatric Illness Scale: 


\begin{tabular}{|c|c|c|c|c|c|c|c|c|c|}
\hline & \multicolumn{3}{|c|}{ Beni Suef University students } & \multicolumn{3}{|c|}{ Coiro University students } & \multirow[t]{3}{*}{$\mathbf{P}$} \\
\hline & & & \multirow{2}{*}{$\begin{array}{c}\text { Agree } \\
\%\end{array}$} & \multirow{2}{*}{$\begin{array}{c}\text { Equivocd } \\
\%\end{array}$} & \multirow{2}{*}{$\begin{array}{c}\text { Disagree } \\
\%\end{array}$} & \multirow{2}{*}{$\begin{array}{c}\text { Agree } \\
\%\end{array}$} & \multirow{2}{*}{$\begin{array}{c}\text { Equivocd } \\
\%\end{array}$} & \multirow{2}{*}{$\begin{array}{c}\text { Disagree } \\
\%\end{array}$} & \\
\hline & & & & & & & & & \\
\hline \multirow{10}{*}{ 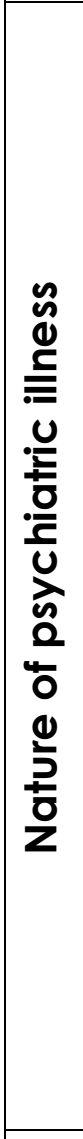 } & Q1 & $\begin{array}{c}\text { Most } \\
\text { dangerous }\end{array}$ & 19.4 & 58.0 & 22.6 & 0.0 & 38.5 & 61.5 & $<.001$ \\
\hline & Q2 & $\begin{array}{l}\text { Dead though } \\
\text { alive }\end{array}$ & 20.5 & 48.6 & 30.9 & 4.6 & 23.1 & 72.3 & $<.001$ \\
\hline & Q3 & Foilure in life & 16.6 & 38.5 & 44.9 & 0.9 & 20.9 & 78.2 & $<.001$ \\
\hline & Q4 & Blessed & 7.4 & 49.8 & 42.8 & 4.7 & 57.1 & 38.2 & .111 \\
\hline & & & & & & & & & N.S \\
\hline & Q5 & Dullards & 21.8 & 39.5 & 38.7 & 0.4 & 39.2 & 60.4 & $<.001$ \\
\hline & Q6 & $\begin{array}{l}\text { Always } \\
\text { aggressive }\end{array}$ & 12.8 & 56.2 & 32.5 & 4.6 & 20.2 & 75.2 & $<.001$ \\
\hline & Q7 & $\begin{array}{l}\text { Hears weird } \\
\text { voices }\end{array}$ & 9.1 & 60.1 & 30.8 & 1.1 & 20.0 & 78.9 & $<.001$ \\
\hline & Q8 & $\begin{array}{c}\text { like mentally } \\
\text { retorded }\end{array}$ & 9.1 & 42.4 & 48.5 & 1.7 & 38.3 & 60 & $<.001$ \\
\hline & Q9 & $\begin{array}{c}\text { Visuad } \\
\text { hallucination }\end{array}$ & 14.8 & 47.3 & 37.9 & 13.9 & 56.2 & 29.9 & $\begin{array}{l}.057 \\
\text { N.S }\end{array}$ \\
\hline \multirow{10}{*}{ 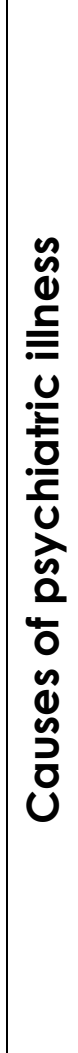 } & Q10 & $\begin{array}{l}\text { Terrestrial } \\
\text { affliction }\end{array}$ & 9.5 & 64.2 & 26.3 & 4.3 & 69.6 & 26.1 & .023 \\
\hline & Q11 & Contagious & 27.9 & 35.8 & 36.3 & 2.3 & 78.8 & 18.9 & $<.001$ \\
\hline & Q12 & $\begin{array}{c}\text { Being } \\
\text { possessed }\end{array}$ & 27.2 & 54.3 & 18.5 & 1.7 & 62.9 & 35.4 & $<.001$ \\
\hline & Q13 & Night quiver & 12.8 & 48.1 & 39.1 & 1.4 & 69.8 & 28.8 & $<.001$ \\
\hline & Q14 & $\begin{array}{l}\text { Decreased } \\
\text { religion }\end{array}$ & 30.9 & 39.9 & 29.2 & 3.75 & 25.0 & 71.3 & $<.001$ \\
\hline & Q15 & Hereditary & 5.4 & 68.7 & 25.9 & 4.2 & 58.5 & 37.3 & .009 \\
\hline & Q16 & $\begin{array}{c}\text { Substance } \\
\text { abuse }\end{array}$ & 20.1 & 42.4 & 37.5 & 5.6 & 59.6 & 34.8 & $<.001$ \\
\hline & Q17 & Physical illness & 6.9 & 43.6 & 49.5 & 3.3 & 58.8 & 37.9 & $<.001$ \\
\hline & Q18 & $\begin{array}{c}\text { Orisis (life } \\
\text { events) }\end{array}$ & 13.5 & 73.3 & 13.2 & 3.7 & 57.1 & 39.1 & $<.001$ \\
\hline & Q19 & Mariage a & 4.1 & 62.2 & 33.7 & 1.9 & 51.2 & 46.9 & .001 \\
\hline
\end{tabular}

Table 4: Distribution of Responses of both groups on each Item of the Beliefs about Psychiatric Illness Scale 
IV- Correlation between Familial Socioeconomic Status Scale and Beliefs about Psychiatric Illness Scale:

\begin{tabular}{|c|c|c|c|c|c|c|c|}
\hline & & $\begin{array}{l}\text { Psychol } \\
\text { ogical } \\
\text { Beliefs } \\
\text { Total }\end{array}$ & $\begin{array}{l}\text { Nature of } \\
\text { psychiatri } \\
\text { c illness }\end{array}$ & $\begin{array}{l}\text { Causes } \\
\text { of } \\
\text { psychiat } \\
\text { ric illness }\end{array}$ & $\begin{array}{l}\text { Psychiatri } \\
\text { c } \\
\text { managem } \\
\text { ent }\end{array}$ & $\begin{array}{l}\text { Psychiatr } \\
\text { ic cure }\end{array}$ & $\begin{array}{l}\text { Effect } \\
\text { on the } \\
\text { family }\end{array}$ \\
\hline \multirow{3}{*}{$\begin{array}{c}\text { Familial } \\
\text { Socioec } \\
\text { onomic } \\
\text { Status } \\
\text { Scale }\end{array}$} & $r$ & .151 & .146 & .224 & -.083 & .017 & .113 \\
\hline & $\mathbf{p}$ & $<.001$ & $<.001$ & $<.001$ & .026 & .642 N.S & .002 \\
\hline & $\mathrm{n}$ & 723 & 723 & 723 & 723 & 723 & 723 \\
\hline \multirow{3}{*}{$\begin{array}{l}\text { Psycholo } \\
\text { gical } \\
\text { Beliefs } \\
\text { Total }\end{array}$} & $r$ & & .872 & .923 & .102 & .642 & .002 \\
\hline & $\mathbf{p}$ & & $<.001$ & $<.001$ & .006 & $<.001$ & $<.001$ \\
\hline & $\mathrm{n}$ & & 723 & 723 & 723 & 723 & 723 \\
\hline \multirow{3}{*}{$\begin{array}{l}\text { Nature } \\
\text { of } \\
\text { psychiatr } \\
\text { ic illness }\end{array}$} & $r$ & & & .890 & -.144 & .444 & .404 \\
\hline & $\mathbf{p}$ & & & $<.001$ & $<.001$ & $<.001$ & $<.001$ \\
\hline & $\mathrm{n}$ & & & 723 & 723 & 723 & 723 \\
\hline \multirow{3}{*}{$\begin{array}{l}\text { Causes } \\
\text { of } \\
\text { psychiatr } \\
\text { ic illness }\end{array}$} & $r$ & & & & -.050 & .529 & .453 \\
\hline & $\mathbf{p}$ & & & & .178 N.S & $<.001$ & $<.001$ \\
\hline & $\mathrm{n}$ & & & & 723 & 723 & 723 \\
\hline \multirow{3}{*}{$\begin{array}{l}\text { Psychiatr } \\
\text { ic } \\
\text { manage } \\
\text { ment }\end{array}$} & $r$ & & & & & .129 & -.152 \\
\hline & $\mathbf{p}$ & & & & & .001 & $<.001$ \\
\hline & $\mathrm{n}$ & & & & & 723 & 723 \\
\hline \multirow{3}{*}{$\begin{array}{l}\text { Psychiatr } \\
\text { ic cure }\end{array}$} & $r$ & & & & & & .247 \\
\hline & $\mathbf{p}$ & & & & & & $<.001$ \\
\hline & $\mathrm{n}$ & & & & & & 723 \\
\hline
\end{tabular}

Table 5: Correlation between Familial Socioeconomic Status Scale and Beliefs about Psychiatric Illness Scale 


\section{Discussion}

In both groups females were significantly more than males $77.8 \%, 65.8 \%$ in Beni Suef University and Cairo University respectively). This could be explained by the fact that faculty of art is predominantly attended by females.

Regarding Familial Socioeconomic Status Scale there was high statistical significant difference between both groups $(p<.001)$ (table 2). The majority of Beni Suef University students were form low and below average class (13.6\%, 49\% respectively). In contrast the majority of Cairo University students were from higher classes (average $41 \%$ and above average $32.5 \%$ ).

Regarding the family history there was statistical significant difference between both groups $(P=.016)$ (table 1). Beni Suef University students had higher family history of psychiatric illness (4.9\%). In contrast the family history among Cairo University students was (1.7\%). This could be explained by Koppel and McGuffin (1999) [13] who found that there was an inverse relationship between socioeconomic status and mental disorders.

Although there was no statistical significant difference between both groups regarding past psychiatric history, we found that it was higher among Beni Suef University students (2.9\% vs. 1\%) (table 1). This could be explained by Chincholikar (2006) [14] finding that psychosocial mal adjustments and psychiatric morbidities were more common among those from lower socioeconomic class.

Cairo University students showed a significantly higher positive attitude regarding

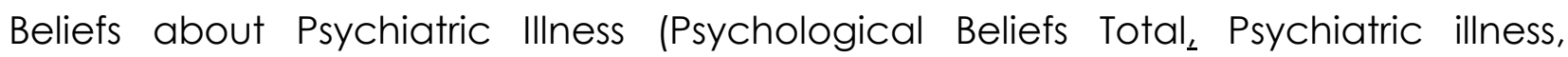
Psychiatric etiology, Psychiatric management, Psychiatric cure, and Effect on the family) ( $p<.001$ ) (table 4). This was in line with Melissa et al., (2004) [15] who found that the most negative answers (negative attitudes) were given by participants who lived in the provinces (lower socioeconomic status), as compared to those living in Athens and the results were statistically significant at the $p<0,001$ level. Also, participants from Athens were willing to accept these people in their social circle. This could be explained by the fact that in poorer areas there is lack of specialized treating facilities and hence disorders tend to be untreated, lack of mental health literacy which leads to feelings of shame, and they suffer more from the burden of the psychiatric illness.

The females in both groups showed lower positive attitude regarding Beliefs about Psychiatric Illness on all subscales but significantly on Psychiatric management, and 
Psychiatric cure ( $p<.001$, and $p=0.001$ respectively) (table 3-b). This was contrary to the finding by Bauman et al., (2003) [16] who reported higher rates of positive attitudes by female students compared to male students. Our results might be explained by the culture difference where in our culture psychiatric disorders is still stigmatizing problem and such problem is highlighted among females which enhance their negative attitude towards psychiatric illness.

Women may suffer from the stigma of mental illness in regard to reduced marital opportunities and increased risk of divorce in an existing marriage, should the condition become public. The higher number of men receiving hospital treatment for schizophrenia in Morocco suggests the stigma is greater for women, as few women come forward for treatment [17].

The students with positive family history in both groups showed a significantly higher positive attitude on beliefs about Psychiatric Illness $(p<.001$ for Psychiatric management, Psychiatric cure, and Effect on the family). Also, there was statistical significant difference between both groups $(p=.034)$ for Nature of psychiatric illness, and $p=.038$ for Causes of psychiatric illness) (table $3-c$ ). This could be explained by the finding that exposure to mental illness generates a more positive attitude. Angermeyer et al., (2004) [18] found that the weaker perceptions of dangerousness corresponded closely with less fear of such people, which in turn was associated with less social distance. Familiarity with mental illness expressed a less strong desire for social distance. This is true even among physicians, Khoweiled (2005) [19] in his study of GPs (over 10 years experience) and House Officers ( $\mathrm{HO}$ ) found that the GP group has a more favorable and more decisive view of mentally ill patients than the $\mathrm{HO}$ group. He explained his finding by the greater acquired knowledge and experience of the GP group who had a minimum of 10 years practice. This is in line with Afana et al. (2000) [20] who showed in their study of the attitude of 166 Palestine primary health care professionals that older professionals showed more emotional and tolerant attitude. They attributed their finding to prior experience with mental illness either through personal or professional activities as Roth et al. (2000) [21] suggested. However Weller \&Grunes (1988) [22] stated that this is not always the case.

The students who have positive past history in both groups showed higher positive attitude on Beliefs about Psychiatric Illness Scale than students who don't have family history. There was high statistical significant difference between both groups $(p<.001$ for Psychiatric cure, Effect on the family, and $P=.001$ for Psychiatric management). Also, there was statistical significant difference between both groups $(\mathrm{P}=.030$ for Nature of psychiatric illness, and $\mathrm{P}=.010$ for Causes of psychiatric illness) (table 3-d). This could be explained by Vezzoli et al., (2007) [23] who stated that 
psychiatric patients had more positive attitude towards mental illness (i.e., less fear, more integration and work opportunity).

Cairo University students showed higher positive attitude on Nature of psychiatric illness. There was high statistical significant difference between both groups $(\mathrm{p}<.001)$ regarding Most dangerous, Dead though alive, Failure in life, Dullards, Always aggressive, Hears weird voices, and Like mentally retarded (table 4). This is contrary to Melissa et al., (2004) [15] who reported that participants who lived in Athens thought that these patients were violent and dangerous to a greater proportion than those who lived in the provinces $(p<0,001)$. However it seems to be in line with the results reported by Moussa and Lawendi (2004) [24] where 48\% of the respondents (all females) from a low socioeconomic area believed that persons with psychiatric illness are the most dangerous in the society and $52 \%$ believed they are always aggressive, which is unfortunately a frequently replicated stereotype of people with mental illness.

Cairo University students showed higher positive attitude regarding causes of psychiatric illness. There was high statistical significant difference between both groups $(p<.001)$ regarding Contagious, Being possessed, Night quiver, Decreased religion, Substance abuse, Physical illness, Crisis (life events), Marriage a related problem, and Skin disease (table 4). Also, There was statistical significant difference between both groups regarding Terrestrial affliction, and Hereditary (table 4). This was consistent with Kabir et al., (2004) [25] found that psychiatric illnesses were caused by drug misuse including alcohol, cannabis, and other street drugs was identified in $34.3 \%$ of the responses as a major cause of mental illness, followed by divine wrath/ God's will (19\%), and magic/spirit possession (18.0\%). Also, Aghanwa (2004) [26] reported that a majority of subjects attributed the cause of mental illness to substance abuse in rural areas in Fiji $(p<0,001)$.

Cairo University students showed higher positive attitude regarding Psychiatric management. There was high statistical significant difference between both groups $(\mathrm{p}<.001)$ regarding Visiting sheikhs, Preparing spirits, Reading Quraan, Natural herbs, ECT, Medications and psychotherapy (table 4). The above results were in accordance with Greenley et al., (1987) [27] who found that psychiatric patients used the available mental health services more in higher social environment. Also, Judd et al., (2006) [28] reported that psychiatric patients in rural culture used health services less because of their lower availability and accessibility in such culture.

Cairo University students showed higher positive attitude regarding cure from psychiatric illness as reflected by their responses to the statements: Never normal 
again, Hopeless case, Mental hospital is a prison, and Symptomatic not cure, $\mathrm{p}<.001$; Cured but recurs; $\mathrm{p}<.022$ (table 4). This might be partially explained by the socioeconomic status where the attitude tends to be more negative in the lower the socioeconomic status. This view is substantiated by the findings reported by Moussa \& Lawendi (2004) [24] who conducted their study in a very low socioeconomic area in Cairo where even a higher percentage of respondents reported a negative attitude: $64 \%$ agreed that the illness is recurrent, $62 \%$ viewed mental hospitals as prisons and $52 \%$ were of the view that medications are tranquillizing rather than curative.

This was consistent with Melissa et al., (2004) [15] who found that majority of participants from higher socioeconomic areas stated that psychiatric patient could be cured, get an education and return to normal life $(p<0,001)$.

Cairo University students showed a significant higher positive attitude regarding Effect on the family $(\mathrm{p}<.001)$. (Tarnishes family's reputation, Burden on family, Tarnishes girl's reputation at marriage, Tarnishes male's reputation at marriage, His death is better, Patient should marry a patient, and Patient should be isolated by his family) (table 4). This was consistent with Aghanwa (2004) [26] who reported that majority of subjects in rural areas in Fiji were less willing to marry or employ mentally ill persons. Melissa et al., (2004) [15] also reported that the participants of the provinces as compared to those of Athens, supported the fact that having a spouse with a mental health disorder could be cause for divorce.

There was a highly significant positive correlation between socioeconomic status and Psychological Beliefs Total $(P<.001)$, Nature of psychiatric illness $(P<.001)$, Causes of psychiatric illness ( $<<.001$ ), and Effect on the family ( $P=0.002)$ (table 5). This means that the higher socioeconomic status the more positive attitude. This confirms Dietrich et al., (2005) [29] conclusion that respondents in countries with less developed mental health care systems showed less positive attitude, and more frequently relied on helping sources outside the mental health sector and on traditional "alternative" treatment methods. Also, Bauman et al., (2003) [16] found that higher rates of positive attitudes were reported by high SES compared to rural/low SES students in Australia.

There was a significant correlation between Psychological Beliefs Total and Nature of psychiatric illness, Causes of psychiatric illness, Effect on the family $(p<.001)$ and Psychiatric management $(p=.006)$ (table 5) with positive correlation between them. This means that the higher total positive attitude the more positive attitude towards 
illness, etiology, management, cures, and effect on the family. That is to say this tool is highly valid to assess different aspects of beliefs about mental illness.

There was a significant positive correlation between Nature of psychiatric illness and Causes of psychiatric illness, Psychiatric management, and Effect on the family (P $<.001$ ) (table 5). This means that the higher positive attitude towards illness is associated with more positive attitude towards etiology, management, cure, and effect on the family. Nguyen and Degotardi (2003) [30] claimed that values and beliefs about mental illness influence care-seeking behavior, treatment outcomes, effect of illness and even determine the way psychiatry is practiced.

There was a highly significant positive correlation between Causes of psychiatric illness and Psychiatric cure, and Effect on the family ( $<.001$ ) (table 5). This means that the higher positive attitude towards etiology of psychiatric illness is associated with more positive attitude towards cure, and effect on the family. Link et al., (1999) [31] claimed that majority of respondents reported multicausal explanations combining stressful circumstances with biologic and genetic factors. Also, they showed more positive attitude. This explain the more favorable attitude towards psychiatric cure, and effect on the family.

There was a highly significant positive correlation between Psychiatric management and Psychiatric cure, and Effect on the family $(P<.001)$ (table 5). This means that the higher positive attitude towards management of psychiatric illness is associated with more positive attitude towards cure, and effect on the family. Melissa et al., (2004) [15] stated that the majority of the participants who showed positive attitude believed that patients with a psychiatric disease could be cured.

There was a highly significant positive correlation between Psychiatric cure and Effect on the family ( $P<.001$ ) (table 5$)$. This means that the higher positive attitude towards psychiatric cure is associated with more positive attitude towards effect on the family. Naeem et al., (2006) [32] reported that positive attitudes towards mental illness is highly associated with increased treatability and recovery. This is associated with minimal burden on the family.

Overall, our findings are important because the more positive attitudes towards psychiatric illness continue to be an important goal for mental and public health professionals. Although the generalizability of our findings may be limited by our reliance on student participants, it is likely that college students are in fact both more comfortable with mental illness and more concerned about social desirability than the general population. We are planning to measure attitudes of the general 
population towards mental illness. Future research should assess stigma associated with a wider variety of predictors and disorders.

\section{Conclusions and Recommendations}

1- Beliefs about mental illness are highly affected by culture difference and socioeconomic status.

2- Public education about psychiatric illnesses is highly needed.

3- A better understanding of mental disorders among the public would allay fear and mistrust about mentally ill persons in the community as well as lessen stigmatization towards such persons.

4- Our findings may be of utility to health policy makers in the design of community mental health education programs taking into consideration the different needs of different strata in Egypt.

\section{References}

1-WHO. Mental health: New understanding; new hope WHO, Geneva. The World Health Report 2001.

2- WHO. Mental health care in developing countries: a critical appraisal of research findings. Report of a WHO Study Group.

World Health Organ Tech Rep Ser. 1984, 698:5-3.

3- Bhugra D. Attitudes towards mental illness. A review of the literature [review]. Acta Psychiatr Scand. 1989, 80:1-12.

4-Wolff G, Pathare S, Craig T, Leff J. Community attitudes to mental illness.Br J Psychiatry. 1996, 168:183-90.

5- Faberga $\mathrm{H}$ J. Psychiatric stigma in non-western societies. Comprehensive Psychiatry. 1991, 32(6):534-51.

6- Asuni T, Schoenberg F, Swift C. Mental health and disease in Africa. Spectrum Books Ltd. 2 Edition Ibadan. 1994, 42-53. 
7- Al- Krenawi A, Graham J R, Ophir M \& Kandah J: Ethnic and gender differences in mental health utilization: The case of Muslim Jordanian and Moroccan Jewish Israeli out-patient psychiatric patients. International Journal of Social Psychiatry. 2001, 47 (3): $42-54$.

8- Corrigan P W, River L P, Lundin R K. Three strategies for changing attributions about severe mental illness. Schizophrenia Bulletin. 2001, 27:187-195.

9- Adebowale T O, Ogunlesi A O. Beliefs and knowledge about aetiology of mental illness among Nigerian psychiatric patients and their relatives.

Afr J Med Med Sci. 1999, 28(1-2):35-41.

10- El-Islam M F. Social psychiatry and the impact of religion. In : Okasha A, Maj M (eds). Images in Psychiatry: An Arab Perspective. Cairo: Scientific Book House, 2001.

11- El-Shakhs A.E. Familial Socioeconomic Status Scale. Anglo Library. 2nd edition (1995).

12- Shukair Z.M. Attitudes Beliefs about Psychiatric Illness in the Arab Culture Scale. Cairo: Alnahda Almasreya Library, (Arabic Reference) 2002.

13- Koppel S, and McGuffin P. Socio-economic factors that predict psychiatric admissions at a local level. Psychological Medicine (1999), 29: 1235-1241.

14- Chincholikar S V. Epidemiological Study of Psychosocial Profile of Blind People. Indian Journal of Community Medicine. 2006, Vol. 31, No. 2.

15- Melissa C,Marvaki C, Gourni M, Tsalkanis A, Pilatis N. People's Attitudes Towards Patients with Mental Illness in Greece. [www.nursing.gr]. 2004, Issue 27.

16- Bauman C, Dupen F, McClellan L, and Vita P. I ncreasing Awareness of Mental illness Among Secondary School Students. An Evaluation of the Mental Illness Education. Centre for Mental Health and Mental Illness Education. Department of Health. 2003, July, 30-85.

17- Kadri N, Manoudi F, Berrada S, Moussaoui D. Stigma impact on Moroccan families of patients with schizophrenia. Canadian Journal of Psychiatry, 2004, 49 (9): 625-629. 
18- Angermeyer M C, Matschinger H, Patrick W. Familiarity with Mental Illness and Social Distance from People with Schizophrenia and Major Depression: Testing A Model Using Data from A Representative Population Survey. Department of Psychiatry, Center for Psychiatric Rehabilitation, University of Chicago. 2004, Volume 69, Issues 2-3, 175-182.

19- Khoweiled A. Attitude of A Sample of Egyptian General Practitioners and Newly Graduated Medical Students Towads Psychiatric Illness. The Egyptian Journal of Mental Health. 2005,42:1-29.

20-Afana A, Dalgard O, Bjertness E, Grunfled B. El-Sarraj E. The attitude of Palestinian primary Healthcare professionals in the Gaza strip towards mental illness, Egyptian Journal of Psychiatry. 2000, 23:101-111.

21- Roth D, Antony HM, Kerr KL, Downie F. Attitudes towards mental illness in medical students: does personal and professional experience with mental illness make a difference? Med Educ. 2000, 34(3): 234-6.

22- Weller I, Grunes S. Does contact with the mentally ill affects nurses attitudes to mental illness? Br J Med Psychol. 1988, 61 (3): 277-84.

23- Vezzoli, R., Archiati, L., Buizza, C., Pasqualetti, P., Rossi, G. Attitude towards psychiatric patients: a pilot study in a northern Italian town. Medical and Societal information from the Research Community TrialStat Corporation. Copyright 2007.

24- Moussa S \& Lawendi M. Mental Health Literacy: A Descriptive Study of a Group of Egyptian Females. Presented at the $6^{\text {th }}$ Regional RCP Meeting, Cairo, Egypt. 2004.

25- Kabir M, lliyasu Z, Isa S, Muktar H. Perception and beliefs about mental illness among adults in Karfi village, northern Nigeria, International Health and Human Rights. 2004, 3-4.

26-Aghanwa H S. Attitude towards and knowledge about mental illness in Fiji Islands. International Journal of Social Psychiatry. 2004 Vol. 50, No. 4, 361-375.

27- Greenley J.R., Mechanic D., \& Clearly P. Seeking help for psychological problems: A replication and extension. Medical Care. (1987), 25, 1113-1128. 
28- Judd F, Cooper A M, Fraser C, Davis J. Rural suicide-people or place effects?. Australian and New Zealand Journal of Psychiatry. 2006, Volume 40 Issue 3 Page 208.

29- Dietrich, S., Matschinger, H. \& Angermeyer, M. C. The relationship between biogenetic causal explanations and social distance toward people with mental disorders. International Journal of Social Psychiatry, in press 2005.

30- Nguyen A, and Degotardi V. Culture and Social Attitudes Towards Mental Illness in Ho Chi Minh City, Vietnam. International Mental Health Cooperation. 2003.

31- Link BG, Phelan JC, Bresnahan M, Stueve A. Public Conceptions of Mental Illness: Labels, Causes, Dangerousness, and Social Distance. Am J Public Health. 1999, 89 (9):1328-33.

32- Naeem F, Ayub M, Javid Z. Stigma and Psychiatric Illness. A Survey of Attitude of Medical Students and Doctors in Lahore, Pakistan. J Ayub Med Coll Abbottabad. 2006,18 (3).

About the authors:

Hani Hamed Dessoki, MD, is Assistant Professor of Psychiatry and Acting Head, Department of Psychiatry at the University of Beni Suef, Egypt. His clinical and research interests include panic and anxiety disorders, psychiatric education, biological psychiatry, familial risk for substance abuse, and women's mental health.

Tamer M.S. Hifnawy MD, is a Lecturer of Public Health, Faculty of Medicine Beni Suif University, one of Upper Egypt Universities. He is an Epidemiologist, conducting researches about Communicable and Non Communicable diseases in Egypt. He is also a teacher of Community Medicine for Undergraduate and post Graduate medical Students.

E-mail: thifnawy@yahoo.com 